\title{
Colloidal Material Box: In-situ Observations of Colloidal Self-Assembly and Liquid Crystal Phase Transitions in Microgravity
}

\author{
WeiBin Li ${ }^{1}$ - Ding Lan' ${ }^{1}$ ZhiBin Sun ${ }^{2}$ BaoMing Geng² XiaoQing Wang $^{2}$. \\ WeiQian Tian ${ }^{1}$ - GuangJie Zhai ${ }^{2}$ - YuRen Wang ${ }^{1}$
}

Received: 14 September 2015 / Accepted: 30 March 2016 / Published online: 25 April 2016

(C) Springer Science+Business Media Dordrecht 2016

\begin{abstract}
To study the self-assembly behavior of colloidal spheres in the solid/liquid interface and elucidate the mechanism of liquid crystal phase transition under microgravity, a Colloidal Material Box (CMB) was designed which consists of three modules: (i) colloidal evaporation experimental module, made up of a sample management unit, an injection management unit and an optical observation unit; (ii) liquid crystal phase transition experimental module, including a sample management unit and an optical observation unit; (iii) electronic control module. The following two experimental plans will be performed inside the CMB aboard the SJ-10 satellite in space. (i) Self-assembly of colloidal spheres (with and without Au shell) induced by droplet evaporation, allowing observation of the dynamic process of the colloidal spheres within the droplet and the change of the droplet outer profile during evaporation; (ii) Phase behavior of $\mathrm{Mg}_{2} \mathrm{Al}$ LDHs suspensions in microgravity. The experimental results will be the first experimental observations of depositing ordered colloidal crystals and their self-assembly behavior under microgravity, and will
\end{abstract}

Ding Lan

landing@imech.ac.cn

YuRen Wang

yurenwang@imech.ac.cn

1 Key Laboratory of Microgravity (National Microgravity Laboratory), Institute of Mechanics, Chinese Academy of Sciences, Beijing 100190, China

2 Key Laboratory of Electronics and Information Technology for Space Systems, National Space Science Center, Chinese Academy of Sciences, Beijing 100190, China illustrate the influence of gravity on liquid crystal phase transition.

Keywords CMB $\cdot$ Microgravity $\cdot$ Self-assembly $\cdot$ Phase transition $\cdot$ SJ-10

\section{Introduction}

Colloidal particle suspensions are ideal model systems to study phase transitions (Zheng et al. ) and defect formation (Cao et al. 2010), because in comparison to atoms, micron-sized colloidal particles are big enough and move sufficiently slowly to allow direct observation with optical microscopy, thus the phase change and defect formation processes on the "atomic" scale could be visualized (Schall et al. 2004). However, larger sized particles settle significantly under gravity, resulting in uneven concentrations of colloidal suspensions. Because phase behavior of colloidal suspensions is closely related to concentration, it is difficult to accurately describe the relationship between the phase transition and the local structure under gravity (Pusey and Van Megen 1986).

Convective assembly is a widely adopted method to form ordered structure in the colloidal suspension (Lin 2012). A Template Assisted Self Assembly (TASA) method was developed by Xia and Yin, which was used to produce a variety of complex aggregates with well-controlled sizes, shapes and structures (Yin et al. 2001). More recently, D.J.Norris explored the properties of photonic crystals by self-assembly from colloidal microspheres, and found that solvent flow played a critical role in controlling the formation process (Norris et al. 2004). Convective assembly relies on the accumulation of particles near three phase contact lines driven by solvent evaporation, which was attributed to 
capillary flow resulting from non-uniform evaporation flux and contact line pinning (Deegan et al. 1997). However, when the convective process induced by evaporation occurs at ground-level, the self-assembly behavior is obscured by gravitational effects including sedimentation and buoyancy convection, and particles are assembled in every desired orientation to the gravitational vector (Hampton et al. 2012; Van Blaaderen et al. 1997). Under microgravity conditions, the self-assembly process induced by evaporation occurs in a uniform environment that is devoid of gravity-driven, deleterious convective mass transport or sedimentation effects (Dag et al. 1997). Therefore, the change to a gravity-free environment is expected to have a remarkable effect on the assembly dynamics and final deposition patterns.

As a colloidal suspension, the phase transition process of liquid crystal is affected by parameters such as gravity, diffusion force and particle dispersion, but the details of these effects remain unclear (Priestly 2012). An theory was formulated to describe the mechanism of phase transition in liquid crystal (Onsager 1949), hypothesizing that the liquid crystal phase transition is an entropy-driven process based on the results of competition, interaction of the excluded volume, and orientational entropy (Frenkel 1987). Onsager theory assumed that the phase transition was an ideal process without consideration of effects of gravity. However, due to the presence of gravity, the diffusion force, polydispersity of particles and long-range interactions between molecules on the ground, the actual phase transition likely differs from the ideal process. Thus, it is important to study the ideal phase transition in microgravity conditions.

Most research demonstrated that gravity plays an prominent role in affecting the structure of colloidal crystals and the phase transition process of colloidal suspensions (Baulin 2003; Murai et al. 2012). The phase behavior of colloidal particles under microgravity conditions has been studied, and experimental results have also been obtained from space research, including nucleation and growth processes in colloidal crystallization (Okubo et al. 1999; Cheng et al. 2001), kinetics of colloidal alloy crystallization of binary mixtures (Ansari et al. 1999; Okubo et al. 2000), and heterogeneous nucleation induced by seed or wall (Schöpe and Wette 2011). The positive influence of gravity on the self-assembly and crystal-growth processes was detected (Dag et al. 1997). Further study of these processes should be performed as space experiments. The recoverable satellite is an effective tool for space experiments in the microgravity environment (Hu 2008; Li et al. 2008), and many space microgravity experiments in China have been completed aboard recoverable satellites since the late 1980's. In the mid-2000s, the Chinese National Space Administration
(CNSA) developed SJ-10 recoverable satellite, which are mainly used to perform experiments of microgravity science and space life science. The engineering phase of program SJ-10 was started by Chinese Academy of Sciences (CAS) since the beginning of 2013, and the satellite is scheduled for launch in early 2016 (Hu et al. 2014), and will stay for 15 days in Earth orbit. The colloidal material box (CMB) is one of several experimental boxes that will be aboard the SJ-10 satellite, which is used to study the self-assembly dynamics of the colloidal spheres (with or without Au-coated nanoparticles) under microgravity, and the phase behavior of liquid crystal in space.

In this paper, we describe the structure and function of the CMB which consists of three modules: (i) a colloidal evaporation experimental module, made up of a sample management unit, an injection management unit, and an optical observation unit, using to observe the self-assembly process of colloidal spheres at the solid/liquid interface and the change of the droplet profile under microgravity conditions; (ii) liquid crystal phase transition experiments module, including a sample management unit and an optical observation unit, to study the phase behavior of liquid crystal in microgravity; (iii) electronic control module, to manage the experimental procedure in Earth orbit and respond to interface of the integrated electrical control box on the satellite.

\section{Scientific Objectives}

\section{Evaporative Self-Assembly Behavior of the Colloidal Spheres at the Solid/Liquid Interface Under Microgravity Conditions}

Previous studies have shown that change in the apparent contact angle with evaporation can lead to change in the flow field of the droplet, and eventually influence the final deposition pattern (Kuncicky and Velev 2008). Under microgravity conditions, the droplet shape changes and tends to be more spherical. Therefore, these experiments can assess the apparent contact angle and its variance with time. We expect to observe the influence of the change of droplet profile on deposition patterns under microgravity. In the process of colloidal droplet evaporation, colloidal spheres were found to accumulate at the solid/liquid interface near three phase contact lines and form ring stain, termed the coffee ring by Deegan and his coworkers. This effect was attributed to edgeward capillary flow induced by the nonuniform evaporation flux of colloidal droplet with pinned contact lines (Deegan et al. 1997). Gravitational effects such as sedimentation and buoyancy flow, which 
Table 1 Space experimental projects in the CMB

\begin{tabular}{|c|c|c|c|c|c|}
\hline $\begin{array}{l}\text { The experimental } \\
\text { projects }\end{array}$ & Samples & Sample types & $\begin{array}{l}\text { Research } \\
\text { content }\end{array}$ & $\begin{array}{l}\text { Experimental } \\
\text { time for each sample }\end{array}$ & $\begin{array}{l}\text { Experimental } \\
\text { methods }\end{array}$ \\
\hline \multirow[t]{2}{*}{$\begin{array}{l}\text { Colloidal } \\
\text { self-assembly }\end{array}$} & $\begin{array}{l}\text { The polystyrene } \\
\text { solution }\end{array}$ & $\begin{array}{l}\text { Volumes }(30,40,50, \\
60,70 \mu \mathrm{L})\end{array}$ & $\begin{array}{l}\text { Colloidal } \\
\text { droplet profile }\end{array}$ & $30 \mathrm{~min}$ & $\begin{array}{l}\text { Side view } \\
\text { images by CCD1 }\end{array}$ \\
\hline & $\begin{array}{l}\text { The polystyrene } \\
\text { solution(spheres } \\
\text { with Au shell) }\end{array}$ & & $\begin{array}{l}\text { Motion of } \\
\text { colloidal } \\
\text { particles }\end{array}$ & $30 \min$ & $\begin{array}{l}\text { Microscopic } \\
\text { images by } \\
\text { CCD2 }\end{array}$ \\
\hline $\begin{array}{l}\text { Liquid } \\
\text { crystal phase } \\
\text { transitions }\end{array}$ & $\begin{array}{l}\mathrm{Mg}_{2} \mathrm{Al} \mathrm{LDHs} \\
\text { (layered double } \\
\text { hydroxide) suspension }\end{array}$ & $\begin{array}{l}6 \text { different } \\
\text { concentrations }\end{array}$ & $\begin{array}{l}\text { Evolution of } \\
\text { liquid crystal } \\
\text { phase }\end{array}$ & 15 days & $\begin{array}{l}\text { Macroscopic } \\
\text { images by } \\
\text { CCD3 }\end{array}$ \\
\hline
\end{tabular}

influence the self-assembly of long-range ordering structure and the formation of defects, cannot be neglected in the convective assembly process (Dag et al. 1997). In the absence of sedimentation and buoyancy convection under microgravity, we will determine the hydrodynamic process of colloidal droplet with evaporation and structure config- uration of deposition patterns, and obtain images of the ordered and disordered phases, previously analyzed on earth (Marín et al. 2011). Our objective is to understand the effect of growth conditions on the colloidal self-assembly process, and improve the growth process for preparation of high quality colloidal crystals.

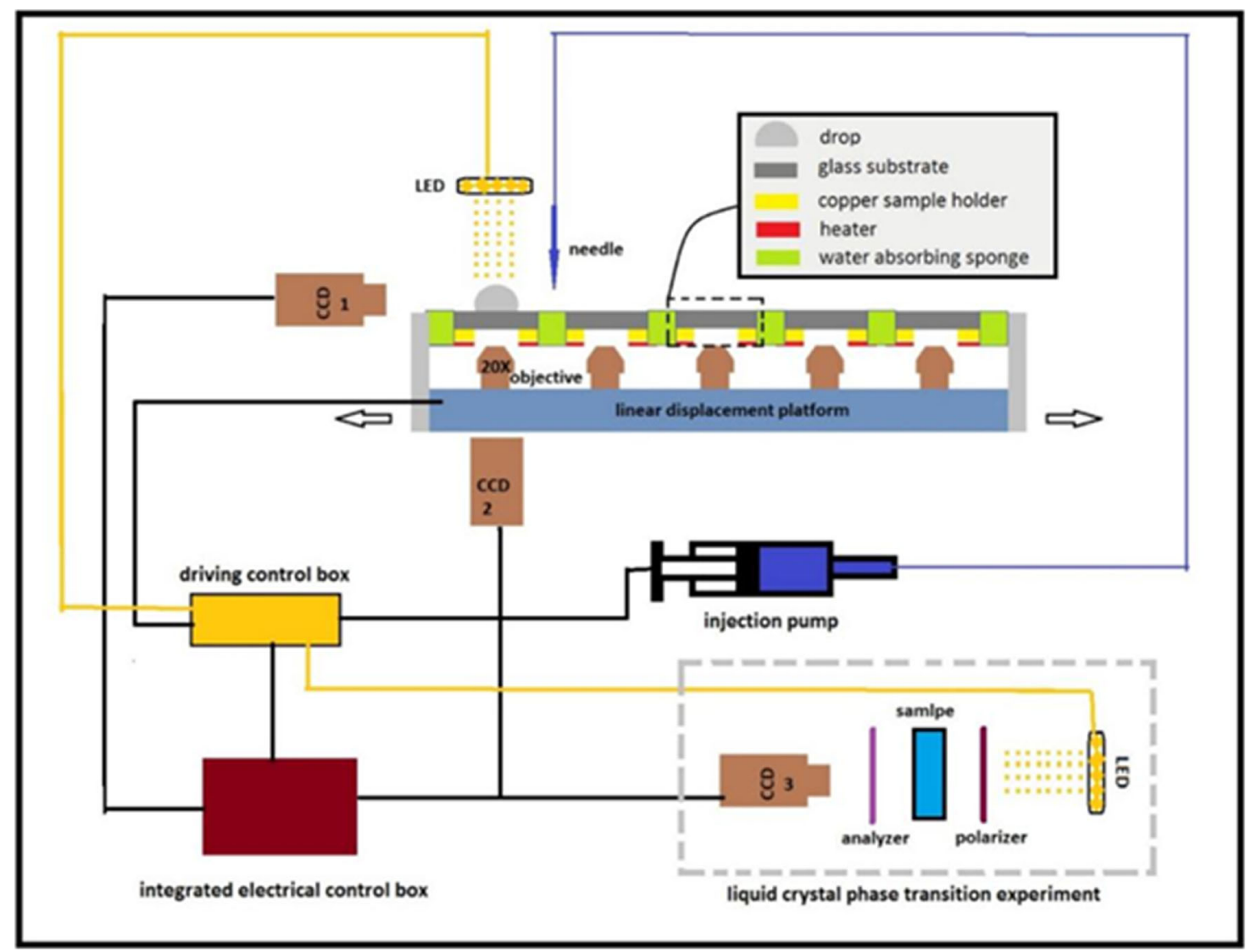

Fig. 1 Experimental principle diagram of the CMB 


\section{Experimental Study of Gravitation Effects on Liquid Crystal Phase Transitions}

Lots of research have shown that liquid crystal formation is highly affected by the gravitational force (Leferink Op Reinink et al. 2012; Leferink op Reinink et al. 2013). The natural sedimentation of particles with different sizes due to gravity causes density gradient and particle size fractionation effect, which undoubtedly facilitate the formation of an ordered phase. Therefore, it is difficult to observe the ideal phase transition process under normal gravity conditions. In this experiment, our studies could give an explanation to the influence of gravity on the liquid crystal phase transition by direct comparison of samples with and without the earth gravitational field. Our aim is to study the phase behavior of liquid crystal driven by entropy alone under microgravity, and illustrate gravitation effects on liquid crystal phase transition.

\section{Design of the Space Experiments}

Two experimental projects to be implemented in space are listed in Table 1. (i) Self-assembly of colloidal spheres induced by evaporation under microgravity conditions. As shown in Fig. 1, five colloidal drops with different volumes will be injected in sequence onto the five sample positions installed on the linear displacement platform. The change of colloidal droplet profile with evaporation will be recorded by CCD1 camera, capturing side-view images at $0.5 \mathrm{fps}$. These images will be used to calculate the apparent contact angle, volume, radius, and height of the droplet. The timedependent contact angle of the droplet will be obtained. In addition, the dynamic deposition process of colloidal microspheres during evaporation will be captured by CCD2 camera at $5 \mathrm{fps}$, in which continual microscopic images will reveal the movement direction and deposition rate of the particles at the solid/liquid interface during droplet evaporation. We will also obtain images of ordered and disorder phases of the final deposition by moving the linear displacement platform. (ii) Phase behavior of $\mathrm{Mg}_{2} \mathrm{Al} \mathrm{LDHs}$ suspensions in microgravity. Six liquid crystal samples of different concentrations will be put in the sample positions within the analyzer and polarizer as shown in Fig. 1. We will observe the phase transition process using CCD3 camera, and obtain color macroscopic images of the liquid crystal phase transition at 30-minute intervals. From the images, we will obtain the phase transition time, the volume fraction of the ordered phase. We will further investigate the alignment of liquid crystal molecules, and the dependence of liquid crystal formation on concentration of the dispersed phase. In addition, through further analysing the differences between

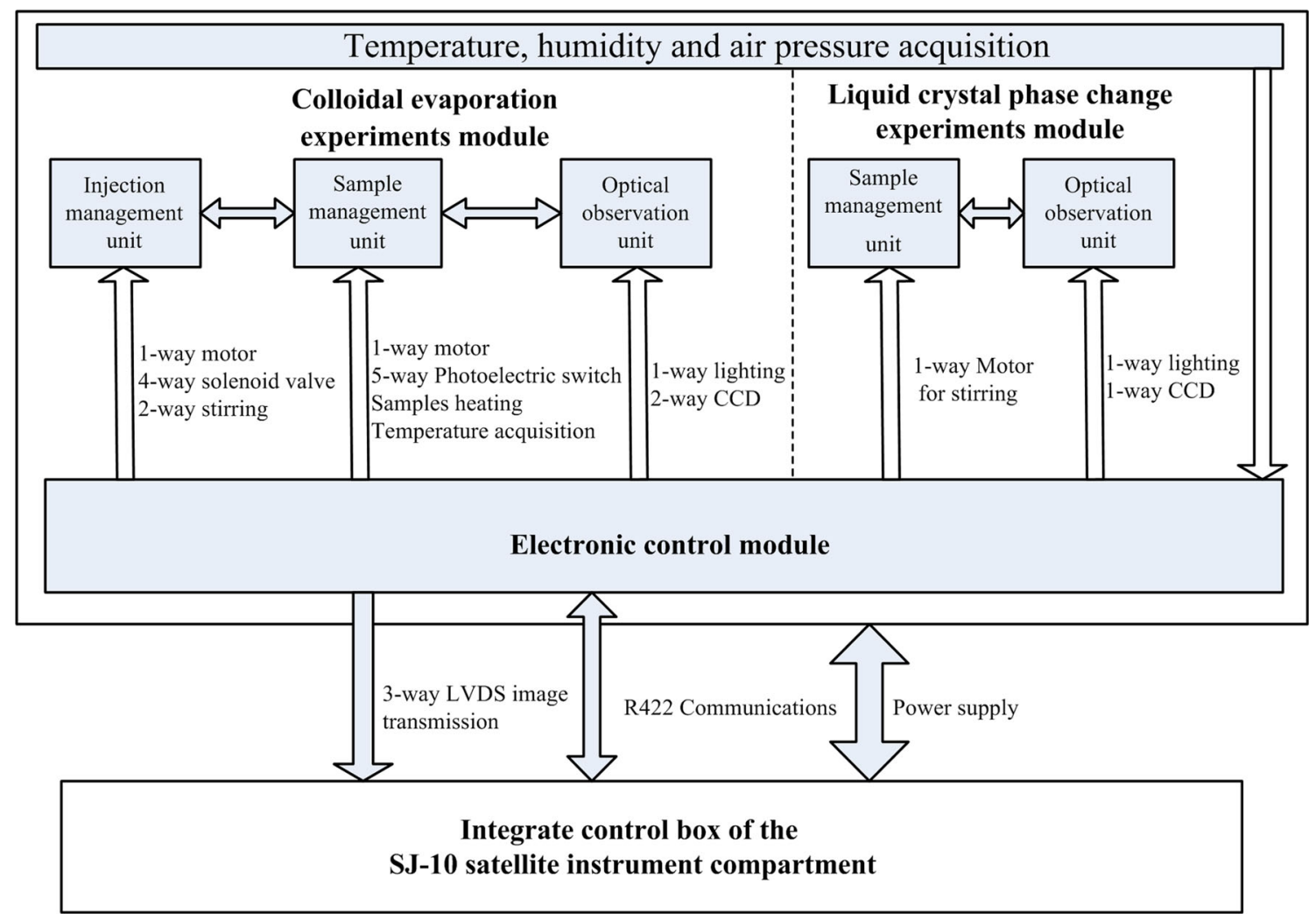

Fig. 2 Flow chart of composition and application of the CMB 
Fig. 3 The internal structure of the CMB

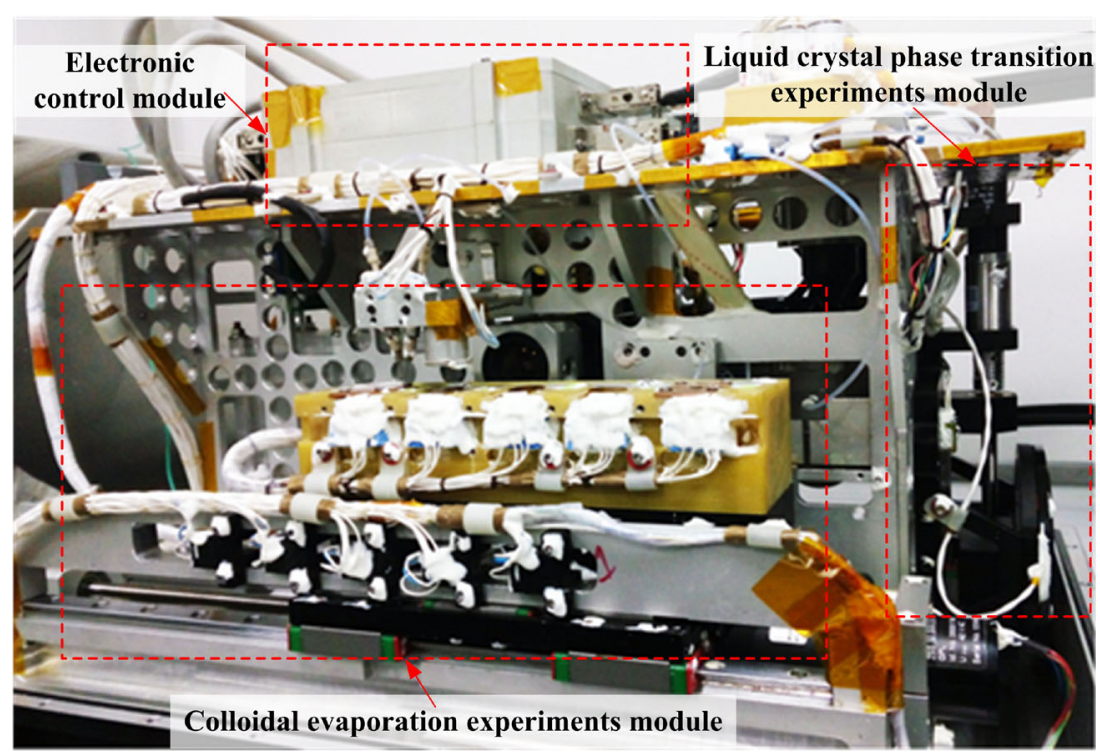

processes performed in space and at ground-level, we will be able to evaluate the gravitation effects on liquid crystal phase transition.

\section{Principle and Structure of the CMB}

The CMB includes three parts as shown in Fig. 2: (i) colloidal evaporation experimental module, composed of a sample management unit, an injection management unit, and an optical observation unit; (ii) liquid crystal phase transition experimental module, which consists of a sample management unit and an optical observation unit; (iii) electronic control module, to manage the experimental procedure of the first two modules and respond to the interface of the integrated electrical control box located in the instrument compartment on the SJ-10 satellite, allowing image acquisition and transmission. Figure 3 shows the internal structure of the CMB.

\section{Colloidal Evaporation Experimental Module}

The sample management unit was designed to provide sample positions necessary for a drop evaporation experiment, heating the samples, and implementing conversion of the sample positions. The optical observation unit will be used to observe the outer droplet profile and the motion of colloidal particles within the droplet during evaporation. These two units were integrated in the colloidal experimental table as shown in Fig. 4a.


Fig. 4 a The colloidal experiments table; $\mathbf{b}$ The structure of a colloidal sample position 


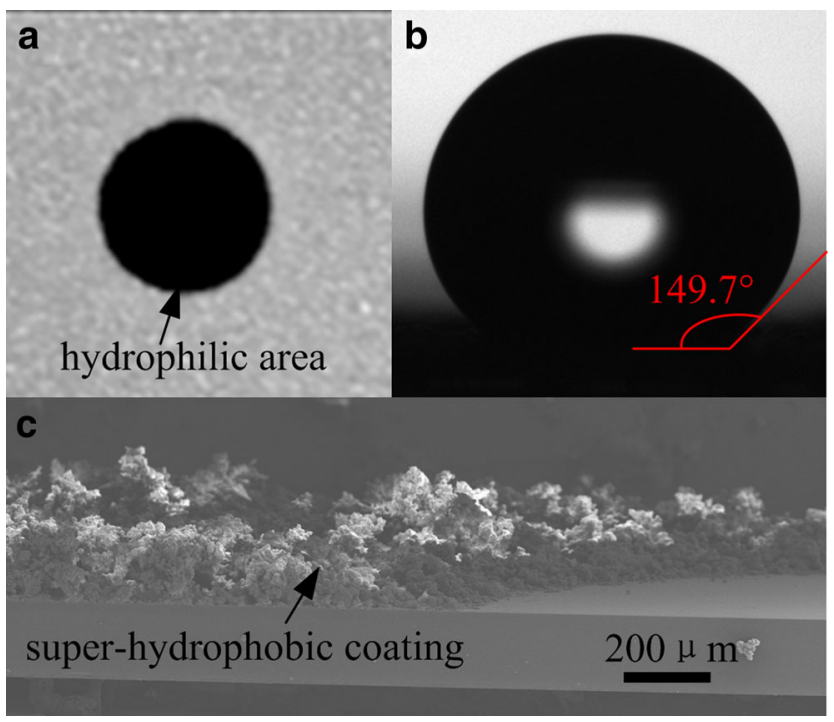

Fig. 5 Substrate with confined hydrophilic area. a A quartz crystal wafer with confined area treated by super-hydrophobic coating, the white part is the coating while the center part is the confined hydrophilic area; $\mathbf{b}$ The super-hydrophobic contact angle of the coating is about $150^{\circ}$; $\mathbf{c}$ SEM image of the cross section of substrate shows that the thickness of the coating is nearly $60 \mu \mathrm{m}$

There are five colloidal sample positions with sample substrates, one motor, a linear displacement platform, sets of parts for sample heating and a thermal sensor (DS18B20), five microscope objectives, one deflective optical path, and two CCDs. The motor can move the linear displacement platform back and forth linearly, and the five sample position can be switched sequentially. Figure $4 \mathrm{~b}$ shows that each sample position has a heating plate. The heating range can be controlled between 40 and 55 degrees Celsius, and the stability of temperature control is less than 1 degrees Celsius. The thermal sensor (DS18B20) contacting the sample substrate is used for temperature acquisition.

The five microscopic objectives were fixed on the linear displacement platform, and each of them was placed just below the sample position, so they could move synchronously with displacement of the platform (Fig. 4b). CCD2 was fixed on the experimental table, which allows observation of microscopic images in the corresponding sample position by deflective optical path, the hole for positioning the optical path, and the axis of CCD2. The sideview CCD (CCD1) was fixed for macroscopic observation of the droplet profile, as shown in Fig. 1.

Since drainage holes (shown in Fig. 4b) and sample positions are arranged alternately, space experiments in all five sample positions can be completed by moving the platform in one direction. The engineering states of the five positions is exactly the same, so the use of samples, image observing, and heating process can be mutual backups, which increases the chance of success for the colloidal evaporation experiments in space.

The sample substrate will be placed on the copper sample holder, composed of hydrophilic and hydrophobic areas. The round hydrophilic area is a quartz surface with a diameter of $5 \mathrm{~mm}$, which is surrounded by a super-hydrophobic coating (SHOS150; Shunytech). As can be seen in Fig. 5a, the white part is the super-hydrophobic coating and the center part is the confined hydrophilic area. Figure $5 \mathrm{~b}$ shows a

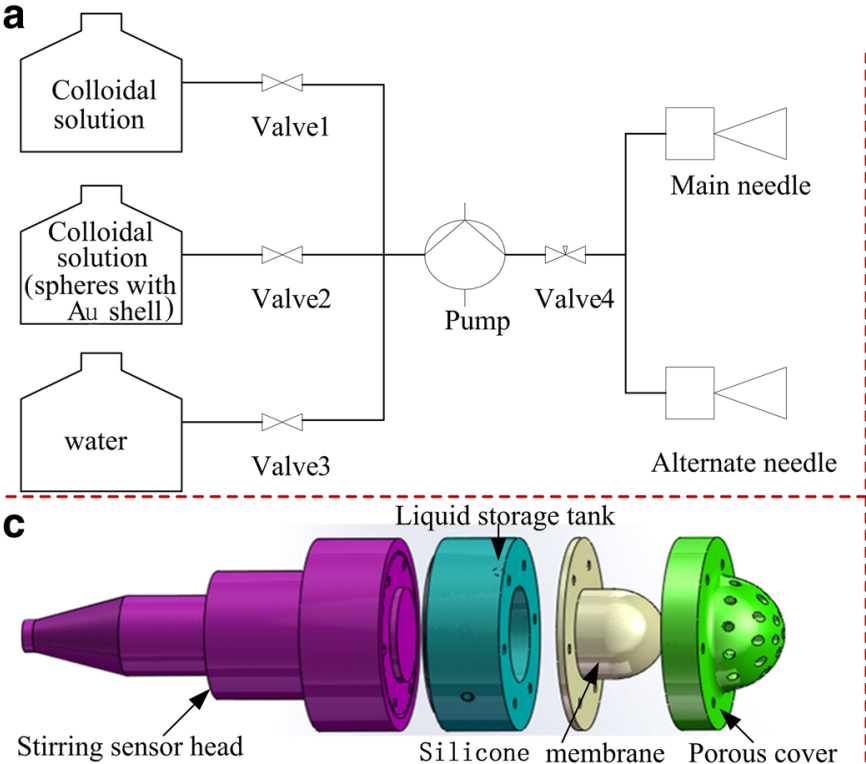

b

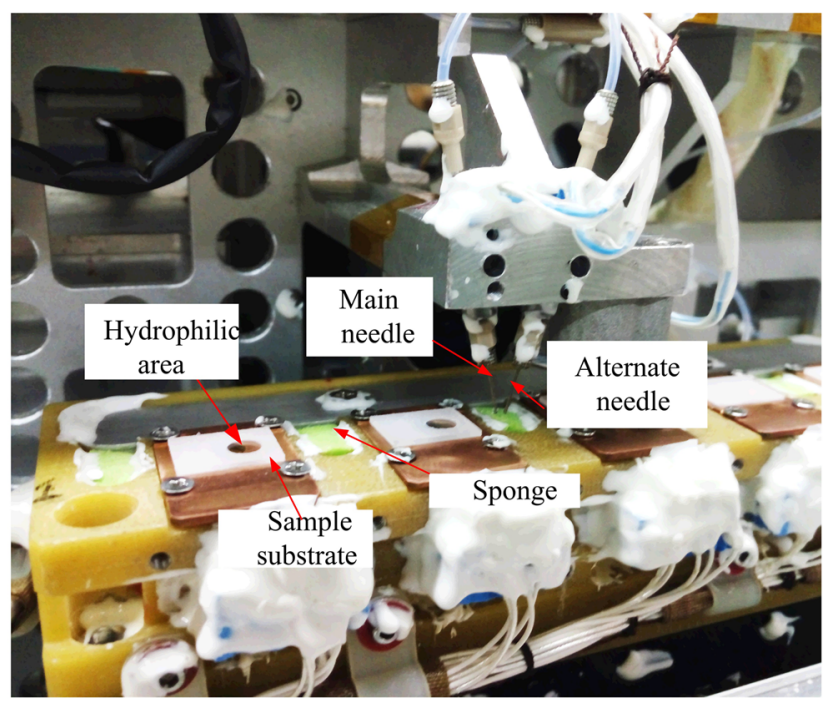

Fig. 6 a Diagram of the injection management unit; b Photos of the injection management unit; $\mathbf{c}$ The schematic of the integrated tank for liquid storage and stirring 
Table 2 The working processes of the injection management unit

\begin{tabular}{|c|c|c|c|c|c|}
\hline Working processes & Pump condition & Valve No.1 & Valve No.2 & Valve No.3 & Valve No.4 \\
\hline Solution Injection & $\mathrm{E}$ & + & - & - & - \\
\hline (spheres without Au shell) & $\mathrm{D}$ & - & - & - & + \\
\hline Solution Injection & $\mathrm{E}$ & - & + & - & - \\
\hline (spheres with Au shell) & $\mathrm{D}$ & - & - & - & + \\
\hline \multirow[t]{2}{*}{ Pipes Cleaning (water) } & $\mathrm{E}$ & - & - & + & - \\
\hline & $\mathrm{D}$ & - & - & - & + \\
\hline
\end{tabular}

(1) "E" and "D" represent liquid extraction and drainage, respectively (2) “+” and "-"represent the valve being opened or closed, respectively

that the super-hydrophobic contact angle of the coating is about $150^{\circ}$, and its thickness is nearly $60 \mu \mathrm{m}$, as shown in Fig. 5c. The sample substrate could confine a droplet with extremely volume of $100 \mu \mathrm{L}$ within the hydrophilic area, and prevent it from floating away in the microgravity of space. The polystyrene colloidal microspheres, dispersed in water with a mean diameter of $3 \mu \mathrm{m}$ were purchased from Duke (5200A). The polystyrene colloidal suspension will be prepared by diluting the original solution to $0.1 \%(\mathrm{w} / \mathrm{w})$ with demonized water.

The injection management unit allows storage and stirring of colloidal solution, injection of colloidal droplet, and releasing of waste solution. It consists of a liquid storage tank and piezoelectric ceramic stirring sensors, as well as an injection pump, four valves, needles and pipes. The principle of the injection management unit is presented in Fig. 6, and allows solution injection and cleaning. As shown in Table 2 and Fig. 6a, solution injection and pipe cleaning occurs by controlling the liquid extraction and drainage of the pump, and by opening and closing of the four valves. The needle is designed for hydrophobic substances, and the distance between the needle and the sample substrate is less than $2 \mathrm{~mm}$. When the droplet from the needle is larger than $2 \mathrm{~mm}$ in diameter, it will contact the hydrophilic area of the sample substrate or the sponge (Fig. $6 \mathrm{~b}$ ), which will ensure that the solution injection and pipes cleaning process function smoothly in space. In case of the main needle failure, injection can be performed with an alternate needle from another channel.

The liquid storage and stirring tank was designed independently to store, mix, and inject the liquid smoothly without producing negative pressure. During the process of extracting liquid, the silicone membrane undergoes an enormous deformation under the effect of air pressure, due to the flexible characteristics of the silicone membrane. The porous cover can protect the silicone membrane, and also allows the external air pressure to act directly on the silicone membrane, as shown in Fig. 6c. This design allows
Fig. 7 a The part and assembly drawings of the liquid crystal experimental table; $\mathbf{b}$ Schematic of the agitation principle of liquid crystal (magnetic particles are nickel spheres of average grain size of $0.5 \mathrm{~mm}$; magnet is $\mathrm{NdFeB}$ permanent magnet) a

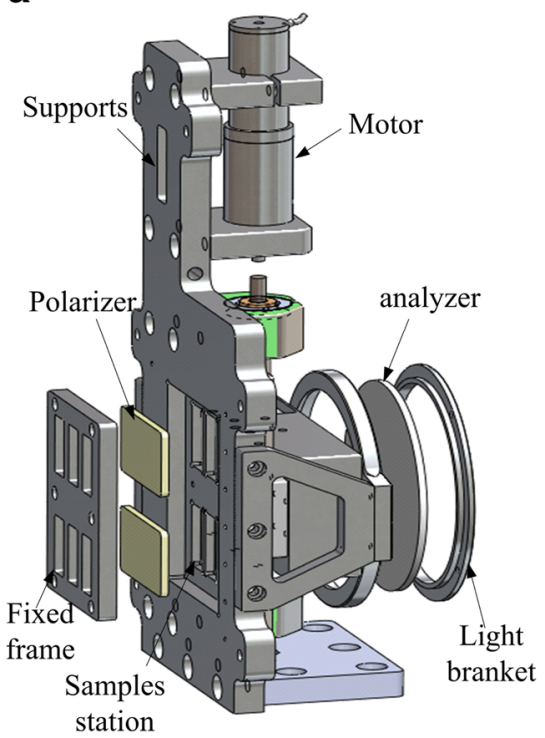

b

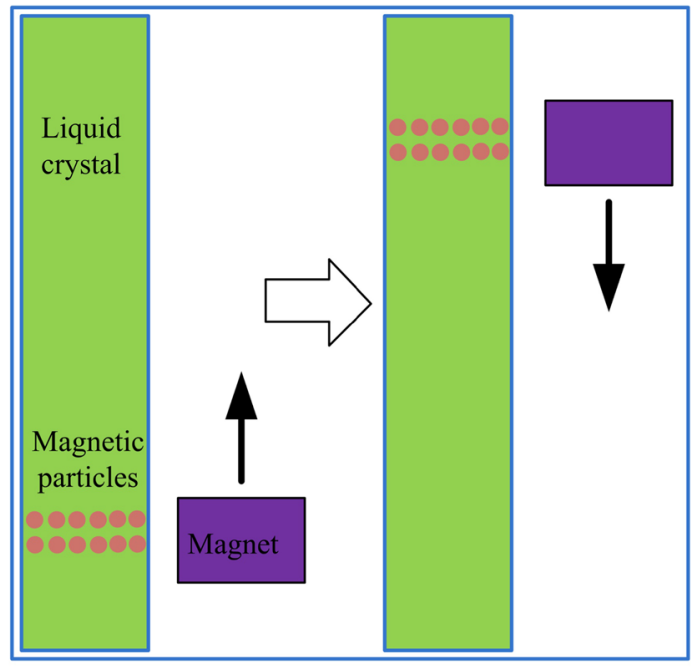


the liquid in the tank to be extracted smoothly both on the ground and the sky. The liquid storage tank and the stirring sensor head were assembled into an integrated tank, which performs ultrasonic stirring of the colloidal solution by high frequency oscillation of the piezoceramics driven by the electronic control box.

\section{Liquid Crystal Phase Transition Experimental Module}

The sample management unit provides liquid crystal sample locations, and can store and agitate the samples. The optical observation unit illuminates the samples and permits observation of the liquid crystal phase transition. These two units were integrated into the liquid crystal experimental table, as shown in Fig. 7a.

The experimental table is made up of six sample locations, supports, one motor, a polarizer, analyzer, fixed frame and a light bracket. There are six different concentrations of the samples which will be put into the corresponding sample locations. To obtain the initial state of liquid crystal solution, the samples must be agitated for an hour. Figure $7 \mathrm{~b}$ shows the agitation principle of liquid crystal. The stepping motor will be controlled to drive the magnet for linear displacement, which drives the magnetic particles inside the sample locations to move due to the magnetic force between them, resulting in liquid crystal mixing.
The observation unit of liquid crystal has a particular optical path (Fig. 7a). The light from the light source passes through the analyzer and polarizer, and the images will be recorded every half an hour by CCD3.

\section{Electronic Control Module}

The electronic control module is performed by the driving control box, its main functions include: switch signal acquisition; control and output of the level or pulse signal of the CCD, LED, colloidal piezoelectric ceramics, solenoid valve, and motor driving signal; output of the PWM signal using in driving the heater chip; RS422 interface communication; and others. It functions as the electronics hardware interface to control how the CMB works. The internal driving control box consists of an analog acquisition and control unit, and driving output unit; the working principle diagram can be seen in Fig. 8.

The driving output unit includes an optoelectronic isolator, transformer isolation circuit, Darlington array driver circuit, D/A conversion circuit and motor driver circuit. The analog acquisition and control unit permit communication with the integrated electronic control box through the RS422 interface. The universal driving control FPGA is located inside the unit, which can control the load operation after the received RS422 instruction is decoded, and return



Fig. 8 The working principle diagram of the driving control box 

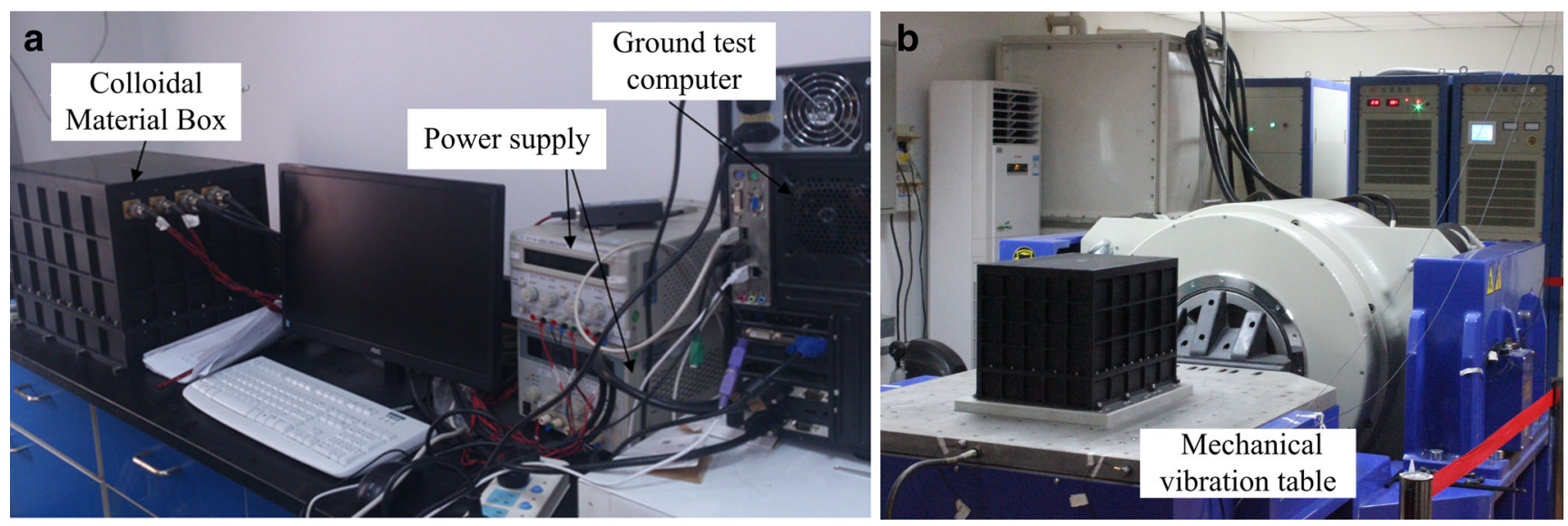

Fig. 9 Photographs of the CMB in ground test

the load state data to the integrated electronic control box for packaging and downlink transfer. For the configuration of the CMB, the universal driving control FPGA can achieve the following functions:

(1) It allows communication of 1-way RS422 asynchronous serial interface link layer;

(2) It can realize analog acquisition of 19 channels;

(3) It can achieve temperature data acquisition of 6-way DS18B20 temperature sensor;

(4) It can obtain 4-way limit switch signals and 6 channel photoelectric switch signals;

(5) It can collect 1-way digital encoder input signal;

(6) It can generate control signals of the three motor driver circuits;

(7) It can control opening and closing of the 2-way piezoelectric actuator;

(8) It can control the three CCD cameras, two LED and 2-way solenoid valve opening and closing;

(9) It can generate the 2-way needle pulse drive signal;

(10) It can calculate 1-way PID and control heating of colloid sample positions by output pulse.

\section{Feasibility Tests of the CMB}

The feasibility tests of the CMB on the ground include the matching test, environmental simulation test and flight simulation test, as shown in Fig. 9. The matching test was first conducted after the product design was completed. The successful operation indicated that the main technical specifications and functions of the CMB were satisfied. The colloidal evaporation and liquid phase transition experiments were conducted and experimental results were as expected. The testing optimization parameters and process flow on-orbit were obtained. The environmental simulation tests, including mechanical tests, a thermal cycle test, a pressure test and a leak test, were conducted to determine cycle life, fatigue life, vibration resistance, temperature limits, and pressure limits. The environmental testing results showed that the CMB could sustain a dynamic environment during satellite launching and withstand the thermal environment once in orbit. The flight simulation tests were carried out after the CMB was installed on the SJ-10 satellite. The entire experimental setup performed remarkably well during simulated fight on the ground, and accomplished 15 days of science operations on-orbit smoothly.

\section{Conclusion}

Our team designed a CMB which consist of three parts: (i) a colloidal evaporation experimental module, used to study the self-assembly behavior of colloidal spheres in the solid/liquid interface, and made up of a sample management unit, an injection management unit and an optical observation unit; (ii) liquid crystal phase transition experimental module, designed to investigate the mechanism of the liquid phase transition under microgravity, and including a sample management unit and an optical observation unit; (iii) an electronic control module, used to manage the experimental procedures during earth orbit and allow communication with the integrated electrical control box.

To accomplish these scientific purposes, two groups of experiments will be conducted inside the CMB aboard the SJ-10 satellite in space. (i) Self-assembly mechanism of colloidal spheres (with and without Au shell) induced by evaporation, including observation of the dynamics of colloidal spheres within the droplet and the change of droplet pro- 
file during evaporation. (ii) Phase behavior of $\mathrm{Mg}_{2} \mathrm{Al} \mathrm{LDHs}$ suspensions in microgravity. The experimental results will allow the deposition of ordered colloidal crystals and study of the self-assembly mechanism under microgravity environment, and will allow illustration of gravitation effects on liquid crystal phase transition.

Acknowledgments Project is supported by National Natural Science Foundation of China (Grant Nos.11202209, 11472275) and Strategic Priority Research Program on Space Science, the Chinese Academy of Sciences (A) (Grant Nos. XDA04020202, XDA04020406).

\section{References}

Ansari, R.R., Hovenac, E.A., Sankaran, S., Koudelka, J.M., Weitz, D.A., Cipelletti, L., Segre, P.N.: Physics of colloids in space experiment. In: Space Technology and Applications International Forum-1999, vol. 1, pp. 108-113 (1999). AIP Publishing

Baulin, V.A.: Self-assembled aggregates in the gravitational field: growth and nematic order. J Chem Phys 119(5), 2874-2885 (2003)

Cao, H., Lan, D., Wang, Y., Volinsky, A.A., Duan, L., Jiang, H.: Fracture of colloidal single-crystal films fabricated by controlled vertical drying deposition. Phys. Rev. E 82(3), 031602 (2010)

Cheng, Z., Zhu, J., Russel, W.B., Meyer, W.V., Chaikin, P.M.: Colloidal hard-sphere crystallization kinetics in microgravity and normal gravity. Appl. Opt. 40(24), 4146-4151 (2001)

Dag, Ö., Ahari, H., Coombs, N., Jiang, T., Aroca-Ouellette, P.P., Petrov, S., Sokolov, I., Verma, A., Vovk, G., Young, D.: Does microgravity influence self-assembly? Adv. Mater. 9(15), 11331149 (1997)

Deegan, R.D., Bakajin, O., Dupont, T.F., Huber, G., Nagel, S.R., Witten, T.A.: Capillary flow as the cause of ring stains from dried liquid drops. Nature 389(6653), 827-829 (1997)

Frenkel, D.: Onsager's spherocylinders revisited. J. Phys. Chem. 91(19), 4912-4916 (1987)

Hampton, M.A., Nguyen, T.A., Nguyen, A.V., Xu, Z.P., Huang, L., Rudolph, V.: Influence of surface orientation on the organization of nanoparticles in drying nanofluid droplets. J. Colloid Interface Sci. 377(1), 456-462 (2012)

$\mathrm{Hu}$, W.: Special issue: microgravity experiments on board the chinese recoverable satellites preface. Springer, New York (2008)

Hu, W., Zhao, J., Long, M., Zhang, X., Liu, Q., Hou, M., Kang, Q., Wang, Y., Xu, S., Kong, W.: Space program SJ-10 of microgravity research. Microgravity Sci. Technol. 26(3), 159-169 (2014)

Kuncicky, D.M., Velev, O.D.: Surface-guided templating of particle assemblies inside drying sessile droplets. Langmuir 24(4), 1371$1380(2008)$
Leferink Op Reinink, A.B.G.M., Van Den Pol, E., Byelov, D.V., Petukhov, A.V., Vroege, G.J.: Ageing in a system of polydisperse goethite boardlike particles showing rich phase behaviour. J. Phys-Condens Mat. 24, 46 (2012)

Leferink op Reinink, A.B.G.M., van den Pol, E., Petukhov, A.V., Vroege, G.J., Lekkerkerker, H.N.W.: Phase behaviour of lyotropic liquid crystals in external fields and confinement. Eur. Phys. J.-Spec. Top. 222(11), 3053-3069 (2013)

Li, C., Zhao, H., Ni, R.: China's recoverable satellites and their onboard experiments. Microgravity Sci. Technol. 20(2), 61-65 (2008)

Lin, Z.: Evaporative self-assembly of ordered complex structures. World Scientific (2012)

Marín, Á.G., Gelderblom, H., Lohse, D., Snoeijer, J.H.: Order-todisorder transition in ring-shaped colloidal stains. Phys. Rev. Lett. 107(8), 085502 (2011)

Murai, M., Okuzono, T., Yamamoto, M., Toyotama, A., Yamanaka, J.: Gravitational compression dynamics of charged colloidal crystals. J. Colloid Interface Sci. 370(1), 39-45 (2012)

Norris, D.J., Arlinghaus, E.G., Meng, L., Heiny, R., Scriven, L.: Opaline photonic crystals: how does self-assembly work? Adv. Mater. 16(16), 1393-1399 (2004)

Okubo, T., Tsuchida, A., Okuda, T., Fujitsuna, K., Ishikawa, M., Morita, T., Tada, T.: Kinetic analyses of colloidal crystallization in microgravity-aircraft experiments. Colloids Surf. A Physicochem. Eng. Asp. 153(1), 515-524 (1999)

Okubo, T., Tsuchida, A., Takahashi, S., Taguchi, K., Ishikawa, M.: Kinetics of colloidal alloy crystallization of binary mixtures of monodispersed polystyrene and/or colloidal silica spheres having different sizes and densities in microgravity using aircraft. Collied Polym. Sci. 278(3), 202-210 (2000)

Onsager, L.: The effects of shape on the interaction of colloidal particles. Ann. NY Acad. Sci. 51(4), 627-659 (1949)

Priestly, E.: Introduction to liquid crystals. Springer Science \& Business Media (2012)

Pusey, P., Van Megen, W.: Phase behaviour of concentrated suspensions of nearly hard colloidal spheres. Nature 320(6060), 340-342 (1986)

Schöpe, H.J., Wette, P.: Seed-and wall-induced heterogeneous nucleation in charged colloidal model systems under microgravity. Phys. Rev. E 83(5), 051405 (2011)

Schall, P., Cohen, I., Weitz, D.A., Spaepen, F.: Visualization of dislocation dynamics in colloidal crystals. Science 305(5692), 1944 1948 (2004)

Van Blaaderen, A., Ruel, R., Wiltzius, P.: Template-directed colloidal crystallization. Nature 385(6614), 321-324 (1997)

Yin, Y., Lu, Y., Gates, B., Xia, Y.: Template-assisted self-assembly: a practical route to complex aggregates of monodispersed colloids with well-defined sizes, shapes, and structures. J. Am. Chem. Soc. 123(36), 8718-8729 (2001)

Zheng, Z., Ni, R., Wang, F., Dijkstra, M., Wang, Y., Han, Y.: Structural signatures of dynamic heterogeneities in monolayers of colloidal ellipsoids. Nat. Commun. 5, 2014 